\title{
Co-Contraction Analyses of the Body Wall Muscles in Caenorhabditis Elegans by Calcium Imaging Assay and Simulation
}

Zu Soh ( $\nabla$ sozu@hiroshima-u.ac.jp )

Hiroshima University

Hiroki Yamashita

Hiroshima University

Michiyo Suzuki

National Institutes for Quantum and Radiological Science and Technology

Kazuma Sakamoto

Hiroshima University

Toshio Tsuji

Hiroshima University

\section{Research Article}

Keywords: locomotion, environments, animals

Posted Date: January 6th, 2021

DOl: https://doi.org/10.21203/rs.3.rs-132897/v1

License: (9) This work is licensed under a Creative Commons Attribution 4.0 International License. Read Full License 


\title{
Co-contraction Analyses of the Body Wall Muscles in Caenorhabditis elegans by Calcium Imaging Assay and Simulation
}

\author{
Zu Soh ${ }^{1, *}$, Hiroki Yamashita ${ }^{1}$, Michiyo Suzuki ${ }^{2}$, Kazuma Sakamoto $^{1}$, and Toshio Tsuji ${ }^{1, *}$ \\ ${ }^{1}$ Department of Electrical, Systems, and Control Engineering, Faculty of Advanced Science and Engineering, \\ Hiroshima University, Higashi-Hiroshima, Hiroshima, 739-8527, Japan \\ ${ }^{2}$ Department of Radiation-Applied Biology Research, Takasaki Advanced Radiation Research Institute, National \\ Institutes for Quantum and Radiological Science and Technology, Takasaki, Gunma, 370-1292, Japan \\ *sozu@hiroshima-u.ac.jp(ZS), tsuji-c@bsys.hiroshima-u.ac.jp
}

\begin{abstract}
Caenorhabditis elegans can generate locomotion under various environments with completely different drag levels. Therefore, animals should have strategies for adapting to the changes in the dynamics of locomotion imposed by various environments. We hypothesized that co-contraction between the ventral and dorsal body wall muscles plays such a role and validated the presence of a co-contraction strategy through both experimental and mathematical modeling approaches. To this end, the fluorescence of calcium ion $\left(\mathrm{Ca}^{2+}\right)$ corresponding to a part of activities of the body wall muscles was measured. The results indicated a significant difference in the co-fluorescence rate between the animals moving in low- and high-drag environments. The contribution of co-contraction to the dynamics of locomotion was then analysed using a body dynamics model. The simulation results suggested that co-contraction allows the dominance of body stiffness over viscous drag so that the phase difference between the local curvature of the body and muscle activities can be maintained under different environmental drag levels. Therefore, co-contraction can be an effective strategy for adapting to environmental drag that changes the dynamics of locomotion.
\end{abstract}

\section{Introduction}

The nematode Caenorhabditis elegans (C. elegans) is a small roundworm with a length of approximately $1.0 \mathrm{~mm}$ and radius of $50 \mu \mathrm{m}$. Because of its size, its movement is associated with a low Reynolds number indicating that viscous drag is dominant in the environment ${ }^{1-4}$. In contrast, the body of the animal is dominated by stiffness when the primary source of stiffness is the cuticle that constitutes the outer shell of the body ${ }^{5,6}$ and the internal pressure imposed by the fluid skeletal structure ${ }^{7}$ The locomotion dynamics depending on both body stiffness and viscous drag can be changed drastically because the animal dwells in completely different drag environments ${ }^{8}$, such as compost ${ }^{9}$, spoiled fruits ${ }^{10}$, and exoskeletons of gastropods ${ }^{11}$. Therefore, C.elegans should have strategies for adapting to changes in the dynamics of locomotion imposed by different environments.

To clarify the mechanism of adaptive motion generation in C. elegans, Berri et al. ${ }^{12}$ constructed a mathematical model that integrates a neural circuit and a body dynamics model; they explained the continuous changes in the undulation wavelength of the body responding to viscous drag in an environment ${ }^{4}$. These studies revealed some important strategies for adaptive motion generation ${ }^{13}$. However, most model studies focused on the wave propagation of a body and assumed that motor neurons alternately contract the body wall muscles on the dorsal and ventral sides. In doing so, they ignored co-contraction on both sides of the muscles, which can change the mechanical properties of the body. This may cause the following discrepancy between theoretical analysis and experimental observation. Mathematical analysis by Fang-Yen et al. ${ }^{14}$ predicted a large phase difference between muscle activity and the generated local curvature in a high-viscosity drag environment. However, Butler $e t$ $a l .{ }^{15}$ compared body curvature with muscle activity measured through calcium imaging and reported that the phase difference between the curvature and muscle activity was nearly constant, irrespective of the viscous drag.

To understand the mechanical properties of the body, our group performed an experiment wherein C. elegans moved along an agar plate. We compared three parameters - curvature, angular velocity, and angular acceleration - against muscle activity. Multiple regression analysis revealed that the partial correlation coefficient of the curvature was the dominant explanatory variable ${ }^{16}$. This indicates that stiffness is the dominant factor in locomotion dynamics, even when the animal moves in a viscous environment with a small Reynolds number. Therefore, the discrepancy between the experimental results ${ }^{15}$ and predictions of the mathematical model ${ }^{14}$ suggests that the stiffness of the body may be insufficient to maintain stiffness-dominant locomotion dynamics in an environment with high viscous drag. Regarding this, it reported that muscle tone increases body stiffness ${ }^{17}$. If 

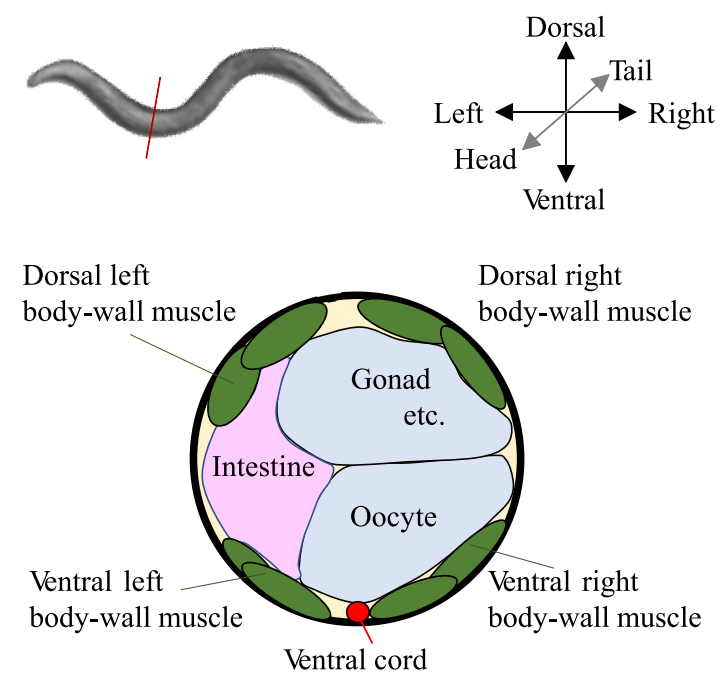

Figure 1. The cross-sectional diagram of the body. The green ellipses correspond to four lines of the body wall muscles extending from the head to the tail.

the animal co-contracts the body wall muscles on both the ventral and dorsal sides (see Fig. 1), the force generated at both the sides can be cancelled, and co-contraction can contribute only toward increasing the body stiffness without interrupting the generation of undulatory motions. Thus, co-contraction can be an effective strategy for maintaining body stiffness.

This study aimed to reveal whether C. elegans employs co-contraction. To this end, we configured low- and high-drag environments to observe the phase differences between muscle activity and the curvature of the animal. Because it is difficult to measure the force exerted by the individual muscle cells directly, we measured muscle activity using a strain that expresses a fluorescent protein on the body wall muscle. In addition, we simulated the motion of $C$. elegans using a body dynamics model incorporated with the measured mechanical property proposed by Cohen's group ${ }^{13,18,19}$ to clarify whether co-contraction contributes toward maintaining body stiffness.

\section{Materials and Methods}

\section{Strains and culture}

Caenorhabditis elegans HBR4:goeIs3[pmyo-3::GCamP3.35::unc-54-3'utrunc-119(+)]V $V^{20}$ and Escherichia coli (E. coli) OP50 strains were obtained from the Caenorhabditis Genetics Center (The University of Minnesota, Minneapolis, MN, USA). The animals were cultured at $20^{\circ} \mathrm{C}^{21}$ on 10 -cm Petri plates (IWAKI non-treated dish, AGC Techno Glass, Shizuoka, Japan) containing with $20 \mathrm{~mL}$ of nematode growth medium (NGM) ${ }^{22}$ on which E. coli was spread and incubated overnight at $37^{\circ} \mathrm{C}$. We used well-fed adult hermaphrodites, synchronously cultured for approximately three days after hatching, in all experiments.

\section{Preparation of drag assays}

The fluorescence intensity of the body wall muscle was measured under free movement conditions on a 6-cm Petri plate (IWAKI non-treated dish, AGC Techno Glass, Shizuoka, Japan) containing with $10 \mathrm{~mL}$ of the NGM. Drag assay was performed 10 days after preparing the NGM plates, with a moisture content of approximately $84 \%$. Hereafter, the surface of the plate is referred to as the low-drag environment. Six to ten animals were picked up from a culture plate using a platina picker (WormStuff Worm Pick, Genesee Scientific Corporation, San Diego, CA, USA) and washed twice in wash buffer ${ }^{23}$ drops on a 6-cm Petri plate without $\mathrm{NGM}^{24}$. The animals were then picked up and transferred to a NGM plate. Subsequently, to evaluate the effects of drag on C. elegans motility, we employed a 2.1-cm square-cut polyethylene terephthalate film (Lumirror film T60125mm, JMT Corporation, Osaka, Japan), $125 \mathrm{mum}$ thick and weighing $79.9 \mathrm{mg}$, and placed it on the NGM plate to cover the animals gently. Hereafter, this environment is referred to as the high-drag environment.

\section{Preparation of antithesis assay}

To measure the fluorescence in the absence of muscle activation, we used an anesthetic. Washed animals were transferred to the NGM plates in the same manner as with the drag assays, and four to six drops of $2 \mathrm{muL}$ of $500 \mathrm{mM}$ sodium azide were placed on the NGM plate to anesthetize the animals ${ }^{25}$. 


\section{Video recording of C.elegans}

To observe the muscular contraction and extension body-wall muscle cells during crawling (forward motion, backward motion, and turn) on an NGM plate, the HBR4 C. elegans, in which reporter gene is used to express the calcium indicator GCaMP3.35 in all body-wall muscle cells, was used. In drag assays under low- and high-drag environments and anesthesia assays, the fluorescence of calcium ion $(\mathrm{Ca} 2+)$ corresponding to muscular contraction in animals was video recorded using a digital camera (High-Speed EXILIM, Casio Computer Co., Ltd, Tokyo, Japan) mounted on a fluorescence stereomicroscope (SZX16, Olympus Corporation, Tokyo, Japan) at 29.97 frames per second and a resolution of $480 \times 640$ pixels. The fluorescence emitted from the body-muscle cells was band-pass filtered at wavelengths of 490 to $560 \mathrm{~nm}$ using a fluorescent filter unit (SZX2-FYFPHQ, Olympus Corporation, Tokyo, Japan). Six or more individuals were video recorded for at least $5 \mathrm{~s}$ in low- and high-drag environments, respectively. For anesthesia assay, five anaesthetized animals were video-recorded for at least $20 \mathrm{~s}$.

\section{Fluorescence intensity measurement}

To measure the fluorescence intensity of the body-wall muscles from the recorded video, we tracked the bodies of the animals. The local fluorescence intensity and curvature were extracted from the fluorescent video. The following describes the measurement procedure.

Step 1: C. elegans tracking

The body of the animal was tracked using worm-tracking software (WormLab, MBF Bioscience, Williston, USA) from the recorded video data, and the tracked body line was equally divided into $N$ points along the body axis. The local curvature of the body was then obtained from the position coordinates of each division point.

Step 2: Extraction of the body range

To determine the body range and measure the fluorescence intensity, the pixel values $Y(x, y)$ at pixel coordinates $(x, y)$ $(x=1,2, \cdots, 640, y=1,2, \cdots, 480)$ were binarised. The pixel set inside the body $\boldsymbol{F}$ and outside the body $\overline{\boldsymbol{F}}$ were determined based on the coordinates of the body outline obtained from the tracking software. The values of the pixels inside the body were then set at $\{Y(x, y)=0 \mid(x, y) \in \boldsymbol{F}\}$, and those outside the body were set at $\{Y(x, y)=1 \mid(x, y) \in \overline{\boldsymbol{F}}\}$. Dilation and erosion was then performed to remove the noise included in $Y(x, y)$.

Step 3: Measuring fluorescence intensity

Let us denote the coordinate vector of a division point $i$ determined in Step 1 as $\boldsymbol{p}_{i}=\left(p_{x, i}, p_{y, i}\right)(i=1,2, \cdots, N)$ (see Fig. 2). The distance $d_{i}$ from the midpoint of the straight line connecting two adjacent points, $\boldsymbol{p}_{i}$ and $\boldsymbol{p}_{i+1}$, to the outside of the body in the direction of the unit vector perpendicular to this straight line was calculated (see the lower left part of Fig. 2).

First, the coordinates $\boldsymbol{h}_{e}=\left\lceil\left(\boldsymbol{p}_{i-1}+\boldsymbol{p}_{i}\right) / 2+e \boldsymbol{v}_{i}\right\rceil$ (where $\lceil\cdot\rceil$ is the ceiling function for a real number) were scanned at increments of $e=1,2, \cdots, E$. The distance from the body axis to the outside of the body was determined as the pixel value changed from $Y\left(\boldsymbol{h}_{e-1}\right)=0$ to $Y\left(\boldsymbol{h}_{e}\right)=1$. If the pixel value of $Y\left(\boldsymbol{h}_{E}\right)=1$ was not detected after scanning approximately twice the body width $(E=30)$, or $Y\left(\boldsymbol{h}_{0}\right)=1$ when $e=1$, the video data were considered inappropriate and excluded from analysis.

Based on the measured distance $d_{i}$, we define the squares $\boldsymbol{A}_{i} \boldsymbol{B}_{i} \boldsymbol{C}_{i} \boldsymbol{D}_{i}\left(\boldsymbol{A}_{i}=\left\lceil\left(\boldsymbol{p}_{i}+\boldsymbol{p}_{i-1}\right) / 2\right\rceil, \boldsymbol{B}_{i}=\left\lceil\boldsymbol{A}_{i}+d_{i} \boldsymbol{v}_{i}\right\rceil, \boldsymbol{C}_{i}=\right.$ $\left.\left\lceil\boldsymbol{D}_{i}+d_{i} \boldsymbol{v}_{i+1}\right\rceil, \boldsymbol{D}_{i}=\left\lceil\left(\boldsymbol{p}_{i}+\boldsymbol{p}_{i+1}\right) / 2\right\rceil\right)$ as shown in the lower right corner of Fig. 4. The fluorescence intensity $G(\boldsymbol{P})$ at the coordinates inside the square $\boldsymbol{P}=\left(x^{\prime}, y^{\prime}\right)$ were obtained, and the average fluorescence value inside the square was obtained. Here, $k=\mathrm{v}$ and $\mathrm{d}$ denote the ventral and dorsal sides, respectively.

The fluorescence intensity is converted to the fluorescence rate using the following equation ${ }^{15}$ :

$$
\bar{G}_{i, t}^{k}=\frac{\bar{G}_{i, t}^{k}-\bar{G}_{i, \min }^{k}}{\bar{G}_{i, \text { min }}^{k}} \quad\left(\bar{G}_{i, \text { min }}^{k}>0\right)
$$

where $\bar{G}_{i, \text { min }}^{k}$ represents the average fluorescence intensity in the absence of muscle activation under anaesthesia. Finally, for each division point $i$, the co-fluorescence component $G_{\mathrm{I}, i, t}$ and the difference component $G_{\mathrm{A}, i, t}$ of the fluorescence rate are calculated as follows:

$$
\begin{aligned}
& G_{\mathrm{I}, i, t}=\min \left({\overline{G^{\prime}}}_{i, t}^{\mathrm{v}},{\overline{G^{\prime}}}_{i, t}^{\mathrm{d}}\right) \\
& G_{\mathrm{A}, i, t}=\bar{G}_{i, t}^{\prime \mathrm{v}}-{\overline{G^{\prime}}}_{i, t}^{\mathrm{d}}
\end{aligned}
$$


A

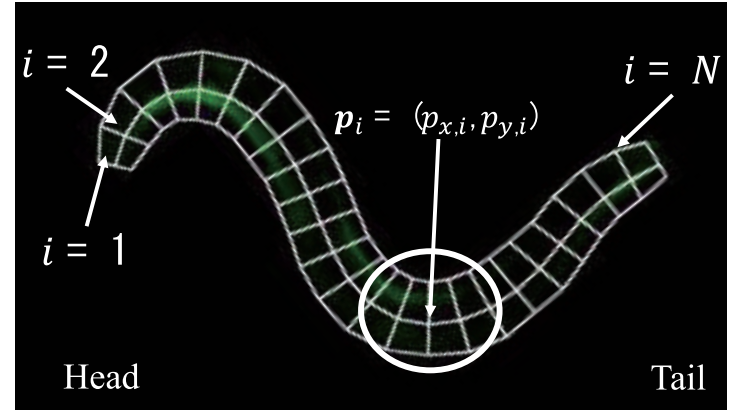

B
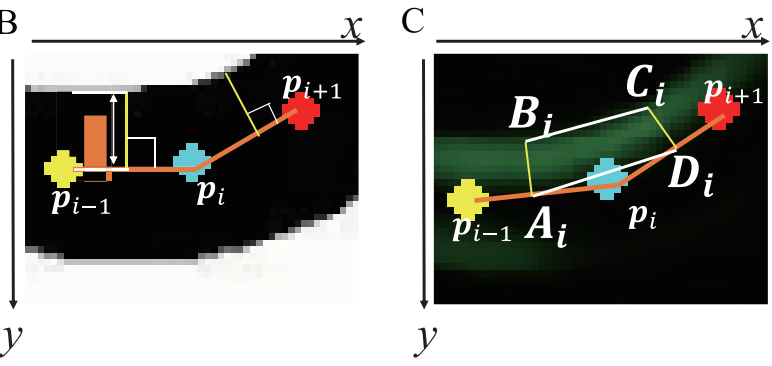

Figure 2. Measuring the distance $d_{i}$ and the definition of the quadrangle in the fluorescence image.

\section{Simulation}

To clarify the effect of co-contraction on the motion of the animal, we simulated the motion using a body dynamics model proposed by Cohen's group ${ }^{4,13}$. We used the $\mathrm{C}++$ code provided by Izquierdo et al. ${ }^{19}$. The body dynamics model is composed of 51 dorsal and ventral pairs of points (50 segments) that approximate the body shape. The points are mutually connected to each other with muscles, viscosity, and stiffness elements. The mechanical parameters were derived from the experimental data. The body dynamics model was driven by muscle activity. The motion was generated by solving the motion equation between force exerted by the muscles and drag of the environment.

We provided muscle activation $F_{j}^{k}$ with the co-contraction component using the following equation, considering that the movement of $C$. elegans was periodic and that the decays of undulation amplitude from the head to the tail.

$$
\begin{gathered}
F_{j}^{d}=\max \left(\frac{A}{\log (j+1)} \sin \left(2 \pi f t-\phi_{j}\right)+b, b\right) \\
F_{j}^{v}=\max \left(\frac{A}{\log (j+1)} \sin \left(2 \pi f t-\phi_{j}+\pi\right)+b, b\right)
\end{gathered}
$$

where $A=0.48 \mathrm{rad}$ is the amplitude, $f=0.4 \mathrm{~Hz}$ is the frequency, $t$ denotes the elapsed time (in seconds), $\phi_{j}=4 \pi j / 24 \mathrm{rad}$ is the phase difference at segment $j$, and $b$ is the co-contraction component. These parameters were determined by trial and error to approach the motion measured in experiments. The curvature at segment $j$ was calculated from the angular velocity $\omega_{j}$ along with the centerline of the body. The phase difference between the curvature and $F_{j}^{k}$ was evaluated.

Based on the coefficient of drag on the agar surface, the tangential drag coefficient in a low-drag environment was set to $C_{\|}=3.20 \times 10^{-3}$, the normal drag coefficient was set to $C_{\perp}=1.28 \times 10^{-1}$. The drag coefficient in a high-drag environment was set to twice the low-drag environment $\left(C_{\|}=6.40 \times 10^{-3}, C_{\perp}=2.56 \times 10^{-1}\right)$.

To clarify how the phase difference between muscle activity and the curvature changes when a co-contraction component is applied to two drag environments, simulations were performed with different $b$.

\section{Results}




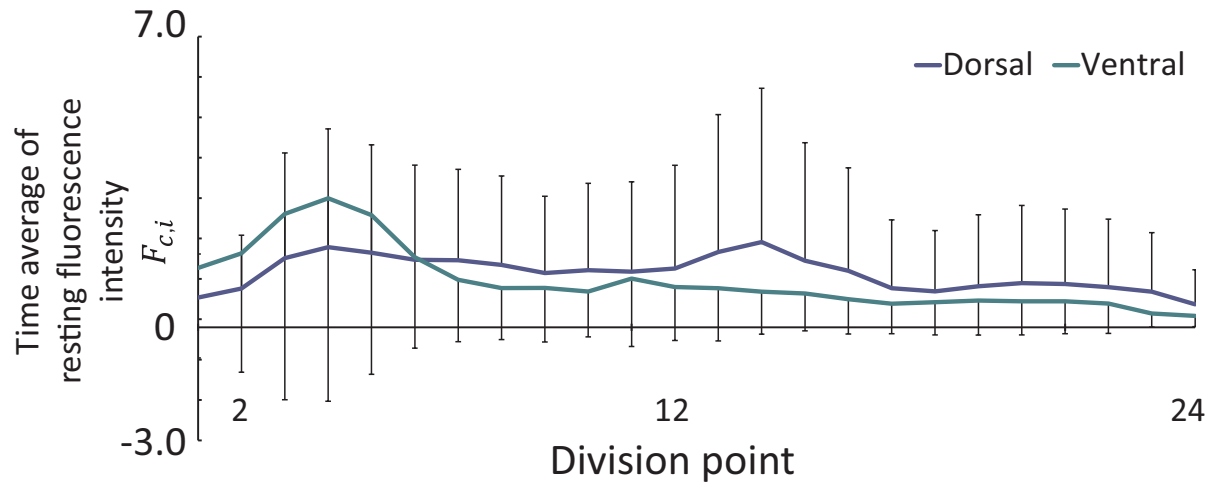

Figure 3. Average fluorescence intensity measured from anaesthetised animals. The $x$-axis denotes the division points, and the $y$-axis represents the fluorescence intensity. The blue and green lines show the individual averages of the fluorescence intensity at the dorsal and ventral sides, respectively. The error bars indicate standard deviations. 
(a)

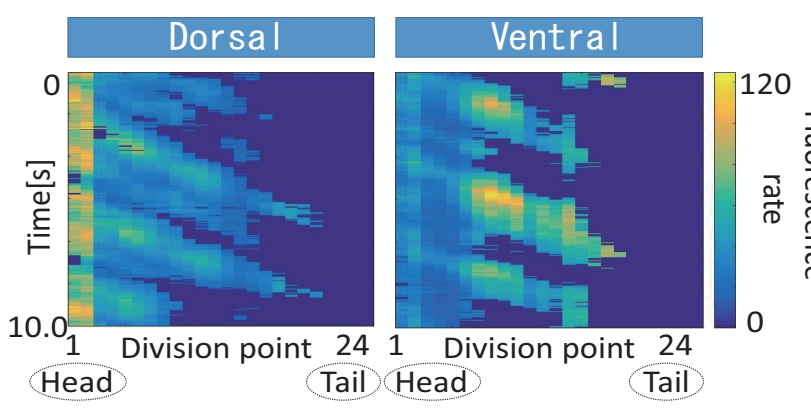

(c)

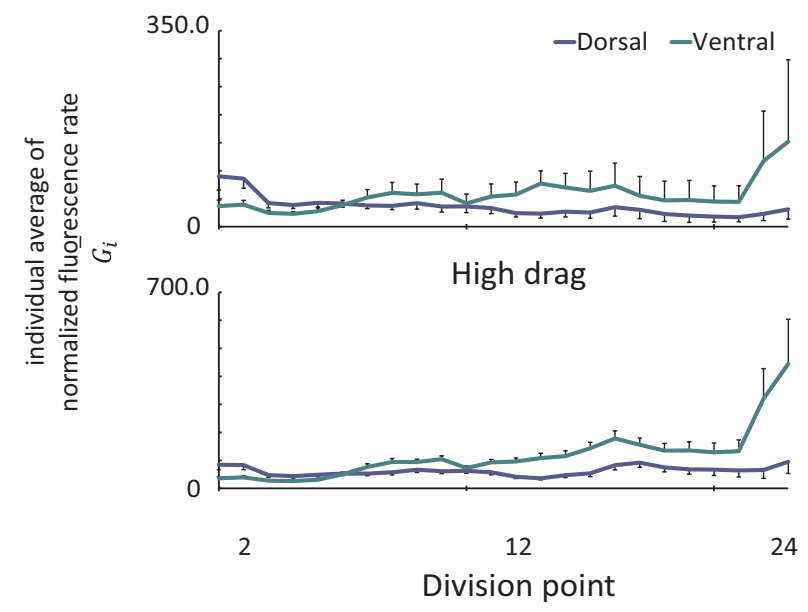

(e)

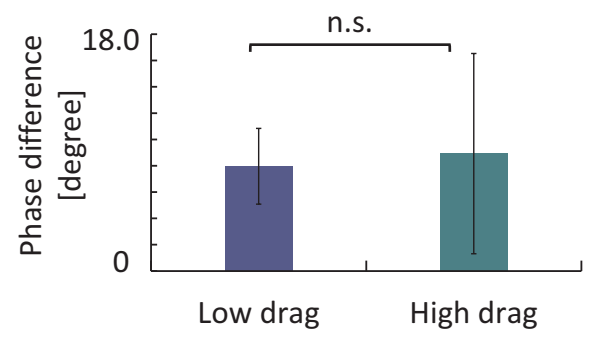

(b)

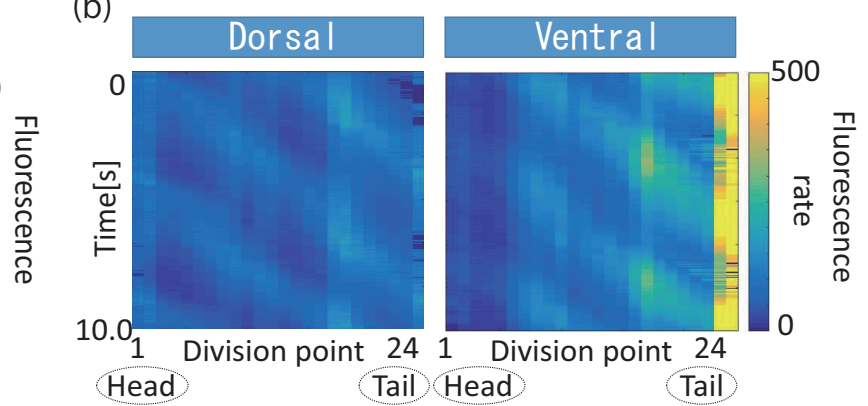

(d)

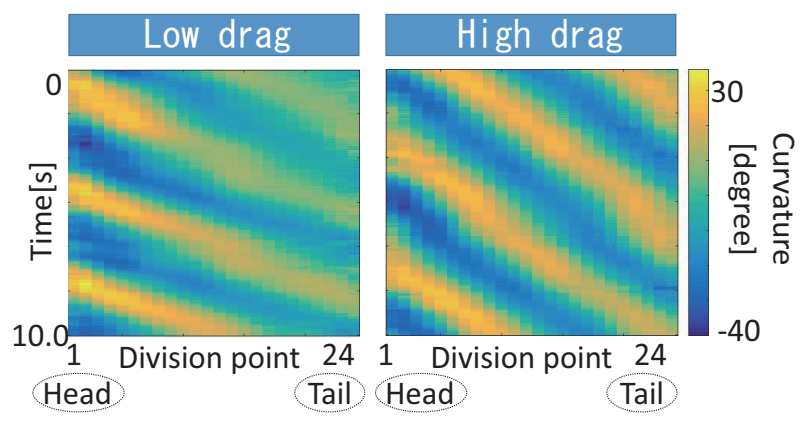

Figure 4. Measured fluorescence rates and curvature. (a) and (b) show examples of the fluorescence rate measured from an individual worm moving in low- and high-drag environments, respectively. The $x$-axis denotes the division points, $y$-axis denotes time, and colour represents the fluorescence rate. (c) shows the fluorescence rate average of all individuals in the lowand high-drag environments at each division point. The $x$-axis denotes the division points, and the $y$-axis is the average fluorescence rate. Blue and green lines indicate dorsal and ventral sides, respectively. (d) shows the curvature of individuals moving through the low- and high-drag environments. (e) shows the phase difference between the curvature and difference components of the fluorescence rate averaged over individuals and division points. 


\section{Negligible effect of environmental drag on the phase difference between local curvature and fluorescence rates}

We measured the fluorescence intensity of the body wall muscles for five individuals paralysed with sodium azide for 306.77 $\mathrm{s}$ to derive the fluorescence intensity in the absence of muscle activity. Fig. 3 shows the average intensity of all individuals calculated for each division point. This result indicates that less fluorescence is emitted from the body wall muscle in the absence of its activation. This average intensity was substituted into $\bar{G}_{i, \min }^{k}$ in Equation (1). All the fluorescence rates shown below were calculated using this $\bar{G}_{i, \min }^{k}$.

We then simultaneously measured the fluorescence rate of the body wall muscle and curvatures for six and nine animals moving freely the in low- and high-drag environments, respectively. The total measurement time in the low-drag environment was $392.89 \mathrm{~s}$, and that in the high-drag environment was $430.63 \mathrm{~s}$. Figs. 4(a) and (b) show $10 \mathrm{~s}$ examples of the fluorescence rates of the individuals moving in the low- and high-drag environments, respectively. Fig. 4(c) shows the average of the fluorescence rates over all individuals in the low- and high-drag environments. A Student's $t$-test comparison of the average fluorescence rate over all individuals and division points indicated that the fluorescence rate of the animal moving in the high-drag environment was significantly higher than that of the animal moving in the low-drag environment $(p<0.05)$.

Fig. 4 (d) shows the curvatures measured from animals in the low- and high-drag environments. The phase difference between the curvature and the difference component of the fluorescence rate $G_{\mathrm{A}, i, t}$ (see Eq.(3)) was then calculated for each division point $i=1,2, \cdots, N=24$. Fig. 4(e) presents the phase differences averaged over individuals and division points. We compared the obtained phase differences between the low- and high-drag environments using a Student's $t$-test and found no significant difference $(p=0.565)$. The average of the phase differences was approximately 9 degrees, which is consistent with that reported earlier ${ }^{15}$. These results confirmed that the environmental drag has little effect on the phase difference between the curvature and difference component of the fluorescence rates.

\section{The environmental drag affects the co-fluorescence component between ventral and dorsal body wall muscles}

We then derived the co-fluorescence component $G_{\mathrm{I}, i, t}$ (see Eq. 2). Fig. 5(a) shows $G_{\mathrm{I}, i, t}$ obtained from each animal over $10 \mathrm{~s}$ moving in the low- and high-drag environments, respectively. The figure indicates that the co-fluorescence component in the high-drag environment is larger than that in the low-drag environment. Fig. 5(b) compares the co-fluorescence components at each division point between low- and high-drag environments using a Student's $t$-test. The comparison showed that at 19 of the $N=24$ division points, the co-fluorescence component in the high-drag environment was significantly larger than that in the low-drag environment $(p<0.05)$. Because the co-fluorescence component reflects co-contraction of the muscles, the above results indicate that the dorsal and ventral body wall muscles co-contract against the drag imposed by the environment. 
(a)

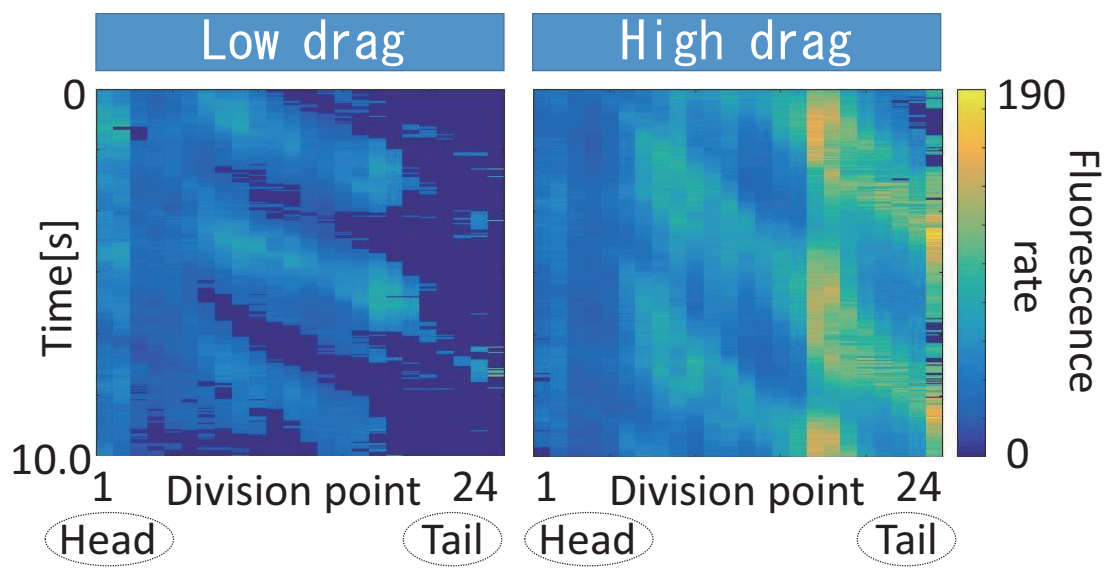

(b)

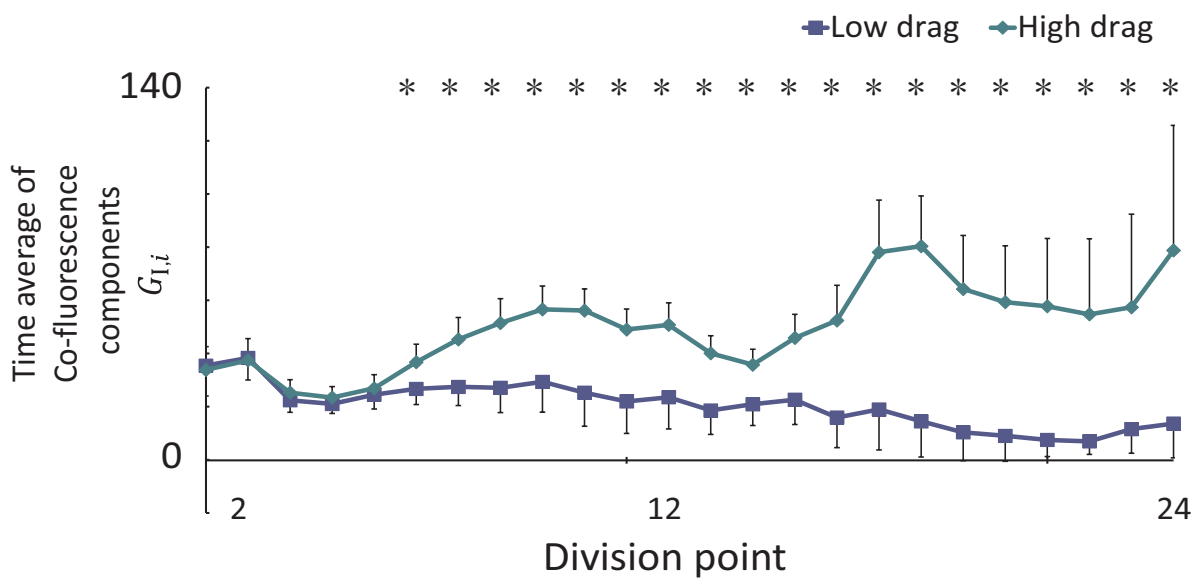

Figure 5. Co-fluorescence component. (a) shows an example of a co-fluorescent component. The $x$-axis denotes division points, $y$-axis denotes time, and colour represents the fluorescence rate. (b) shows the average of the co-fluorescent component over all individuals. The $x$-axis denotes the division points, and the $y$-axis represents the average co-fluorescence rate. 
(a)

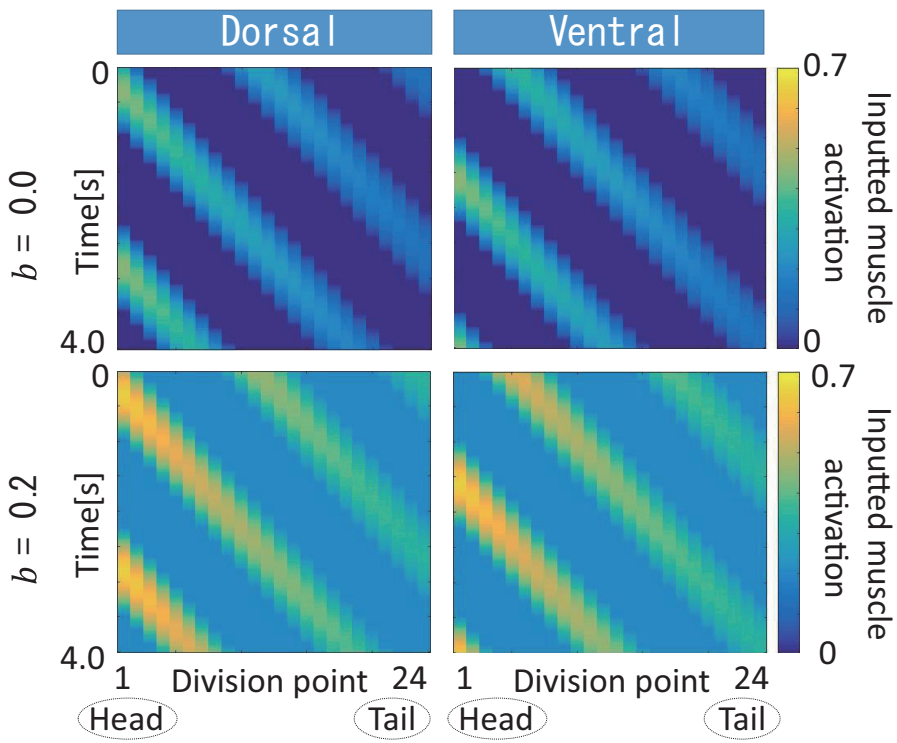

(c)

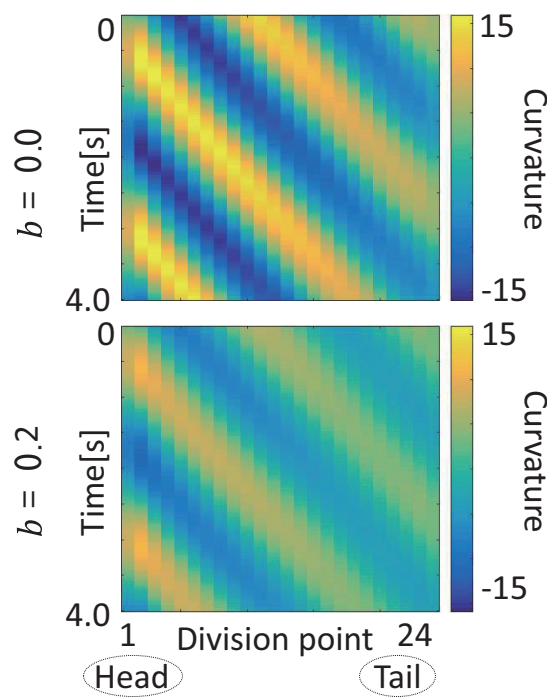

(b)

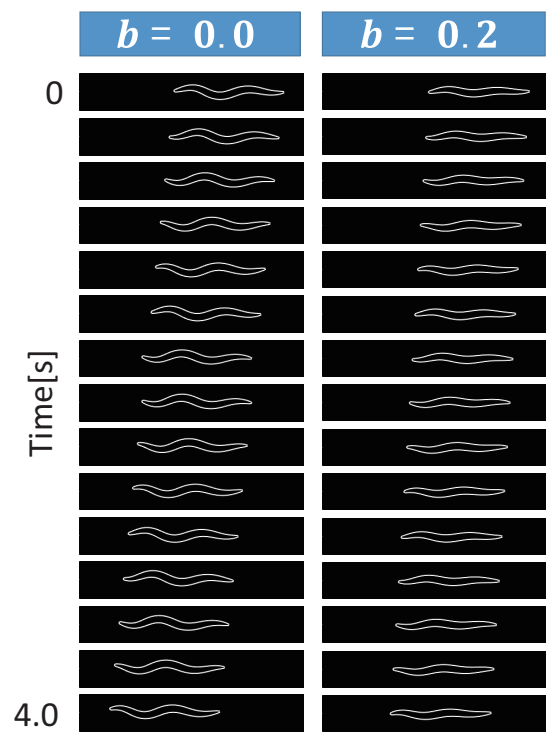

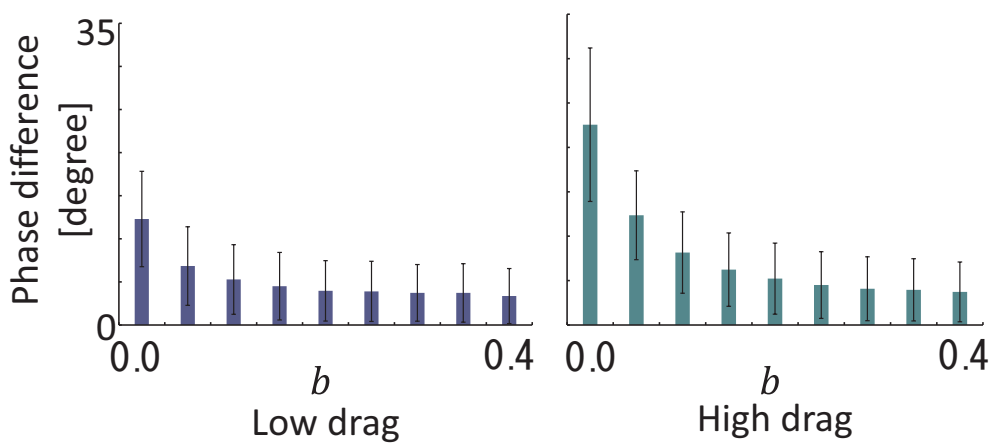

Figure 6. Simulation results of the body dynamics model. (a) shows the input of dorsal and ventral muscle activities with $b=0.0$ and 0.2 . The $x$-axis denotes the division points, $y$-axis represents time, and colour represents the inputted muscle activation. (b) shows the image obtained by sampling the generated motion at equal time intervals with $b=0.0$ and 0.2 . (c) shows the curvature at $b=0.0$ and $b=0.2$. The $x$-axis denotes the division points, $y$-axis indicates time, and colour represents the curvature. (d) shows the individual average of the phase difference between the input muscle activity and curvature when $b=0.0 \cdots 0.4$. The $x$-axis denotes the co-contraction value, and the $y$-axis denotes the phase difference. 


\section{Co-contraction decreases the phase difference between muscle activity and the curvature}

The experimental results presented in the previous section suggest the co-contraction of the dorsal and ventral body wall muscles. However, it is difficult to clarify the effect of co-contraction on locomotion dynamics with this experimental approach. Therefore, we performed a motion generation simulation using a body dynamics model.

Motion was generated by applying the muscle activity defined in Eq. 4 with the co-contraction parameter $b=0.0,0.05, \cdots, 0.4$. Fig. 6(a) shows the applied muscle activation with $b=0.0,0.2$, (b) shows simulated motion driven by the muscle activation under the low-drag environment, and (c) shows the corresponding curvatures. The figures show that the co-contraction component reduces the amplitudes of the curvatures.

We then calculated the phase difference between the inputted muscle activity and the simulated curvature averaged over all division points. Fig. 6(d) shows the resulting phase difference in both the low- and high-drag environments. The phase difference in the low-drag environment without co-contraction $(b=0.0)$ is approximately 12 degrees, which is close to the measured value ${ }^{15}$; however, the phase difference increases to 23 degrees in the high-drag environment. Nevertheless, the co-contraction parameter $b$ can decrease the phase differences, and at approximately $b=0.05,0.1$, the phase difference in the high-drag environment approaches the measured value. These results indicate that co-contraction can cancel the phase difference imposed by the high drag of the environment.

\section{Discussion}

In this study, we analysed whether $C$. elegans employs a co-contraction strategy to counter the effect of drag on locomotion dynamics. Video analysis revealed that the co-fluorescence between the ventral and dorsal sides of the body wall muscles significantly increases against the drag of the environment (see Fig. 5). To understand the effect of co-contraction on the locomotion dynamics, we used a body dynamics model developed by Cohen's group ${ }^{4,13,18,19}$ to simulate motion using muscle activation. The result showed that co-contraction can reduce the phase difference between muscle activity and the local curvature (see Fig. 6). This is because muscle activation increases the stiffness of the body so that the mechanical properties of the body dominate the effect of the viscous drag on the locomotion dynamics. Therefore, co-contraction can be an effective strategy that allows the animal to generate motion under environments with different drag levels.

Previous model studies implicitly assumed that the body wall muscles alternately contract on the dorsal and ventral $\operatorname{sides}^{4,12,26}$. With this assumption, theoretical studies predicted that the phase difference between the body curvature and muscle activity varied considerably, depending on the environment ${ }^{14}$. However, experimental studies showed that the phase difference is consistent under different viscous drags ${ }^{15}$. Therefore, co-contraction strategy may explain this gap between experimental and theoretical studies.

The simulation performed in this study represents co-contraction using bias terms (see Eq. 4), but the co-fluorescence component in the video analysis shows that the co-fluorescence component propagates from the head to the tail (see Fig. 5). Increasing the stiffness along with the local curvature is more energy efficient than simply adding the bias component.

To control co-contraction, it is necessary to obtain drag information from environments. Therefore, co-contraction may be associated with DVA neurons, which can respond to whole body curvature ${ }^{27}$. However, dorsal and ventral motor neurons are connected in a mutually suppressed manner to alternately contract the muscles ${ }^{28}$. Thus, neural-network- level analysis is required to understand how co-contraction is controlled.

\section{Conclusion}

In this study, we measured the activity of the body wall muscles of $C$. elegans using its fluorescence intensity. The analysis revealed the co-fluorescence component of the ventral and dorsal sides of the body wall muscles; this co-fluorescence component increased with environmental drag. Our results suggested the presence of a co-contraction strategy. Through simulations, we confirmed that co-contraction is required to maintain a constant phase difference between muscle activity and the local curvature, irrespective of environmental drag. The energy-efficient control mechanism of the co-contraction strategy requires further investigation involving both experimental and mathematical approaches.

\section{References}

1. Sznitman, J., Shen, X., Sznitman, R. \& Arratia, P. E. Propulsive force measurements and flow behavior of undulatory swimmers at low Reynolds number. Phys. Fluids 22, 121901, DOI: 10.1063/1.3529236 (2010).

2. Sznitman, J., Purohit, P. \& Krajacic, P. Material Properties of Caenorhabditis elegans Swimming at Low Reynolds Number. Biophys. journal 98, 617-626, DOI: 10.1016/j.bpj.2009.11.010 (2010). 
3. Sznitman, J., Shen, X., Purohit, P. K. \& Arratia, P. E. The Effects of Fluid Viscosity on the Kinematics and Material Properties of C. elegans Swimming at Low Reynolds Number. Exp. Mech. 50, 1303-1311, DOI: 10.1007/s11340-010-9339-1 (2010).

4. Boyle, J. H., Berri, S. \& Cohen, N. Gait modulation in c. elegans: an integrated neuromechanical model. Front. computational neuroscience 6, 10 (2012).

5. Zhen, M. \& Samuel, A. D. C. elegans locomotion: small circuits, complex functions. Curr. opinion neurobiology 33, 117-126 (2015).

6. Park, S.-J., Goodman, M. B. \& Pruitt, B. L. Analysis of nematode mechanics by piezoresistive displacement clamp. Proc. Natl. Acad. Sci. 104, 17376-17381 (2007).

7. Niebur, E. \& Erdös, P. Theory of the locomotion of nematodes: control of the somatic motor neurons by interneurons. Math. biosciences 118, 51-82 (1993).

8. Rabets, Y., Backholm, M., Dalnoki-Veress, K. \& Ryu, W. S. Direct measurements of drag forces in c. elegans crawling locomotion. Biophys. J. 107, 1980-1987 (2014).

9. Cutter, A. D. Nucleotide polymorphism and linkage disequilibrium in wild populations of the partial selfer caenorhabditis elegans. Genetics 172, 171-184 (2006).

10. Barrière, A. \& Félix, M.-A. High local genetic diversity and low outcrossing rate in caenorhabditis elegans natural populations. Curr. Biol. 15, 1176-1184 (2005).

11. Wallace, H. Movement of eelworms: V. observations on aphelenchoides ritzema-bosi (schwartz, 1912) steiner, 1932 on florists 'chrysanthemums. Annals Appl. Biol. 47, 350-360 (1959).

12. Berri, S., Boyle, J. H., Tassieri, M., Hope, I. A. \& Cohen, N. Forward locomotion of the nematode c. elegans is achieved through modulation of a single gait. HFSP journal 3, 186-193 (2009).

13. Cohen, N. \& Denham, J. E. Whole animal modeling: piecing together nematode locomotion. Curr. Opin. Syst. Biol. 13, 150-160 (2019).

14. Fang-Yen, C. et al. Biomechanical analysis of gait adaptation in the nematode caenorhabditis elegans. Proc. Natl. Acad. Sci. 107, 20323-20328 (2010).

15. Butler, V. J. et al. A consistent muscle activation strategy underlies crawling and swimming in caenorhabditis elegans. $J$. Royal Soc. Interface 12, 20140963 (2015).

16. Sakamoto, K., Yamashita, H., Soh, Z., Suzuki, M. \& Tsuji, T. Multiple regression analysis to reveal relationships between muscle activities and body bends in a transgenic caenorhabditis elegans. In 26th International Symposium on Artificial Life and Robotics (2021).

17. Petzold, B. C. et al. Caenorhabditis elegans body mechanics are regulated by body wall muscle tone. Biophys. journal 100, 1977-1985 (2011).

18. Boyle, J. H. C. elegans locomotion: an integrated approach. Ph.D. thesis, University of Leeds (2009).

19. Izquierdo, E. J. \& Beer, R. D. From head to tail: a neuromechanical model of forward locomotion in caenorhabditis elegans. Philos. Transactions Royal Soc. B: Biol. Sci. 373, 20170374 (2018).

20. Schwarz, J., Spies, J.-P. \& Bringmann, H. Reduced muscle contraction and a relaxed posture during sleep-like lethargus. In Worm, 1, 12-14 (Taylor \& Francis, 2012).

21. Brenner, S. The genetics of caenorhabditis elegans. Genetics 77, 71-94 (1974).

22. Funayama, T. et al. Heavy-ion microbeam system at jaea-takasaki for microbeam biology. J. radiation research 49, 71-82 (2008).

23. Saeki, S., Yamamoto, M. \& Iino, Y. Plasticity of chemotaxis revealed by paired presentation of a chemoattractant and starvation in the nematode caenorhabditis elegans. J. Exp. Biol. 204, 1757-1764 (2001).

24. Suzuki, M. et al. Development of ultra-thin chips for immobilization of caenorhabditis elegans in microfluidic channels during irradiation and selection of buffer solution to prevent dehydration. J. neuroscience methods 306, 32-37 (2018).

25. Massie, M. R., Lapoczka, E. M., Boggs, K. D., Stine, K. E. \& White, G. E. Exposure to the metabolic inhibitor sodium azide induces stress protein expression and thermotolerance in the nematode caenorhabditis elegans. Cell stress \& chaperones $\mathbf{8}$, 1 (2003). 
26. Suzuki, M., Tsuji, T. \& Ohtake, H. A neuromuscular model of c. elegans with directional control. In Proc. of the first international conference on complex medical engineering, 167-172 (2004).

27. Li, W., Feng, Z., Sternberg, P. W. \& Xu, X. S. A c. elegans stretch receptor neuron revealed by a mechanosensitive trp channel homologue. Nature 440, 684-687 (2006).

28. Sengupta, P. \& Samuel, A. D. Caenorhabditis elegans: a model system for systems neuroscience. Curr. opinion neurobiology 19, 637-643 (2009).

\section{Acknowledgement}

The authors thank the Caenorhabditis Genetic Center for providing strains of C. elegans and E. coli., and members of our laboratories for the valuable discussion. This work was supported by JSPS KAKENHI Grant Number 18K18839 to M.S.

\section{Author Contribution}

H.Y., Z.S., and K.S. performed modelling and simulations, analysed and interpreted the data, and wrote the paper. M.S. and H.Y. performed the behavioural experiments, analysed the data, and revised the paper. T.T. interpreted the simulation data, and critically reviewed the paper. T.T. and Z.S. designed the study, conceived simulations, and revised the paper. All authors provided critical feedback and helped shape the research, analysis, and the paper.

\section{Competing Interests}

K.S. is employee of Sony Corporation. Z. S., M.S., and T.T. declare no potential conflict of interest. 
Figures
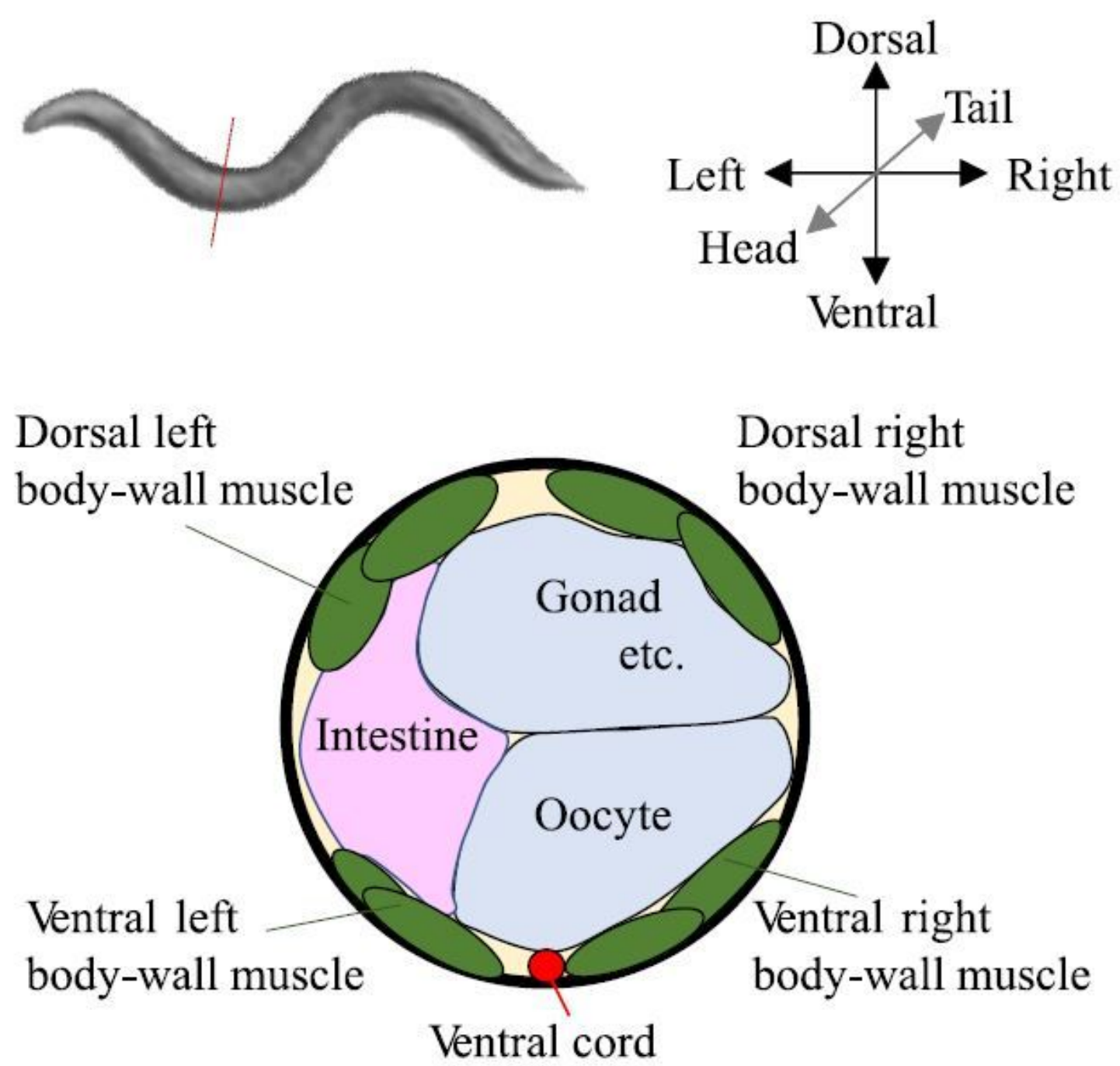

Figure 1

The cross-sectional diagram of the body. The green ellipses correspond to four lines of the body wall muscles extending from the head to the tail. 


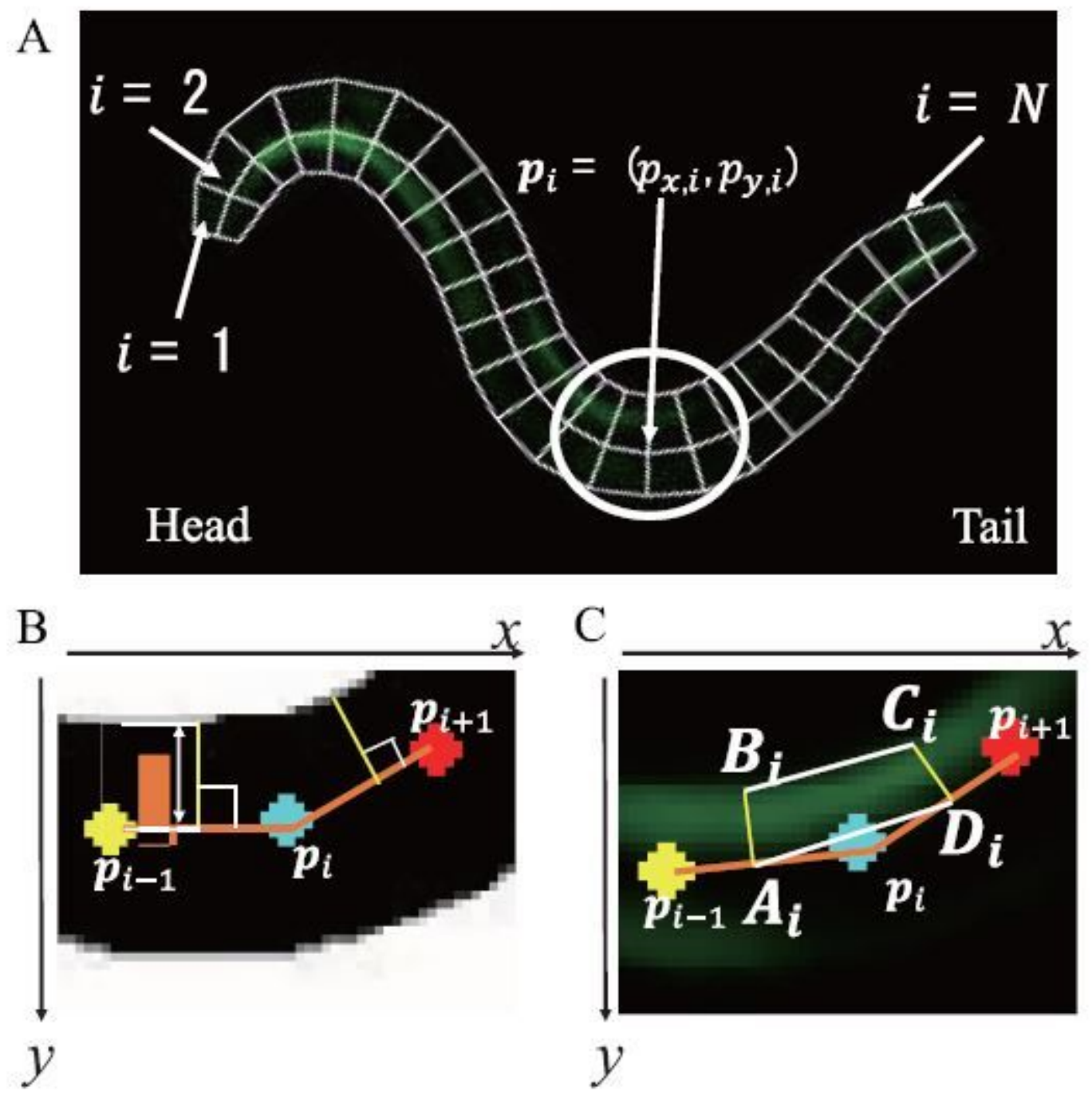

Figure 2

Measuring the distance di and the definition of the quadrangle in the fluorescence image. 


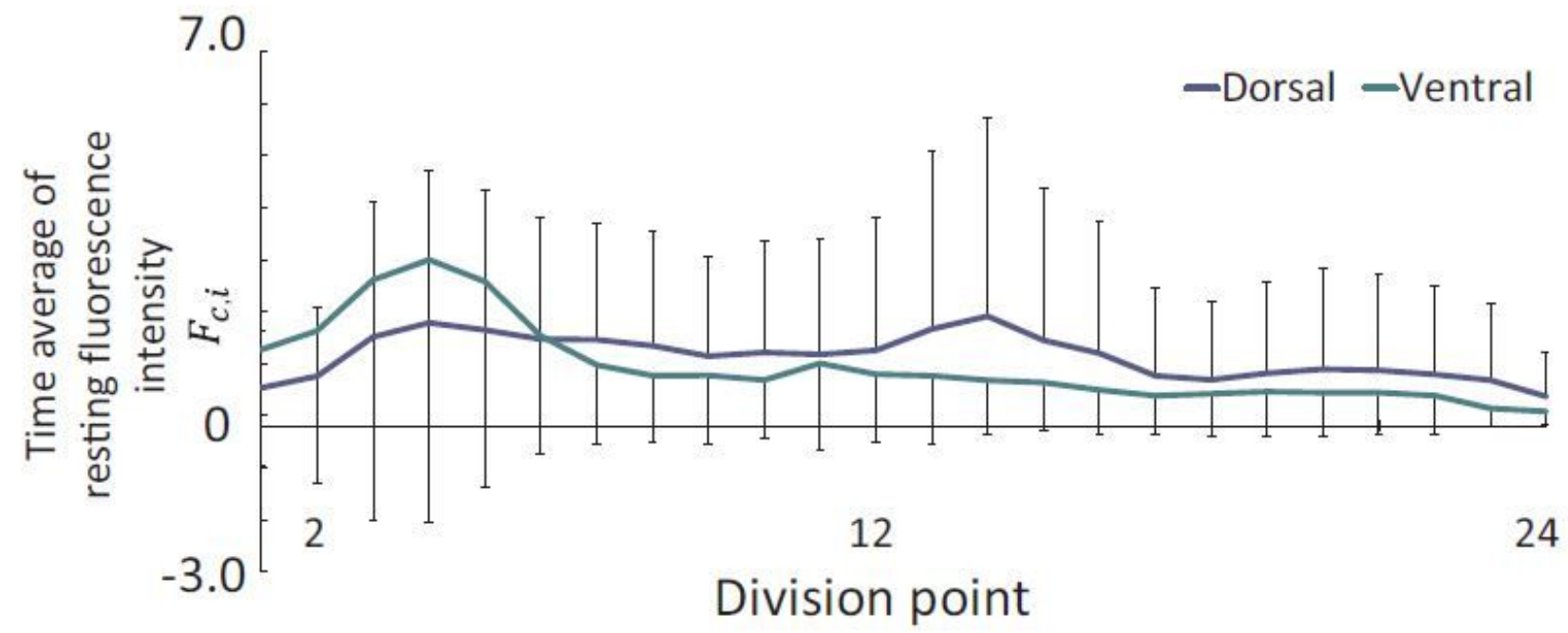

Figure 3

Average fluorescence intensity measured from anaesthetised animals. The x-axis denotes the division points, and the $y$-axis represents the fluorescence intensity. The blue and green lines show the individual averages of the fluorescence intensity at the dorsal and ventral sides, respectively. The error bars indicate standard deviations. 
(a)

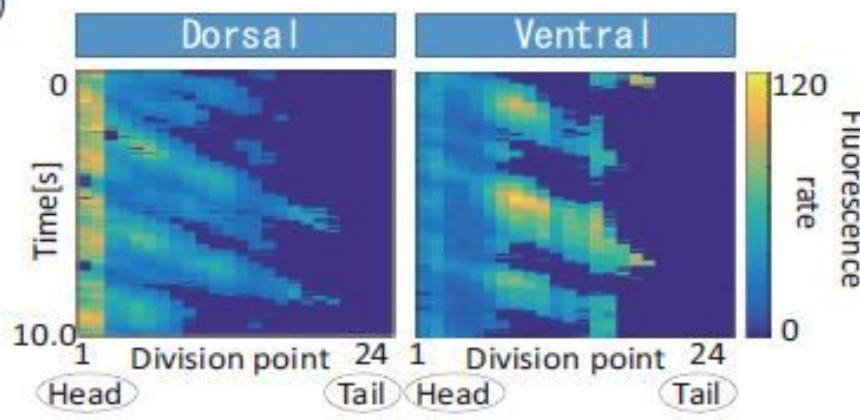

(c)

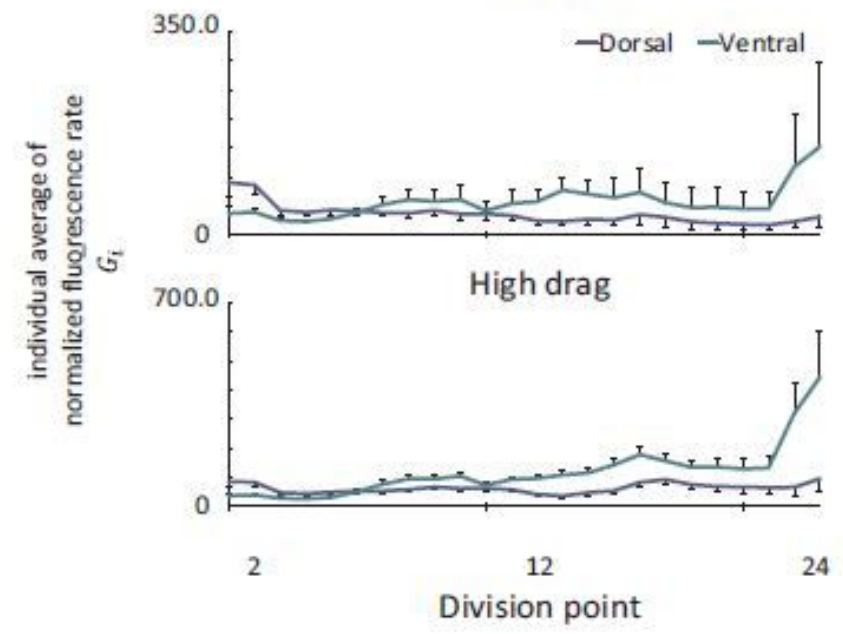

(e)

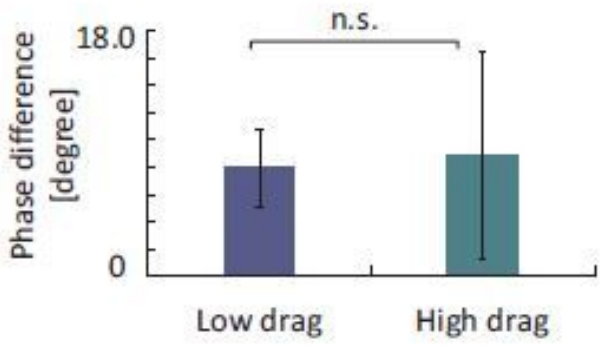

(b)

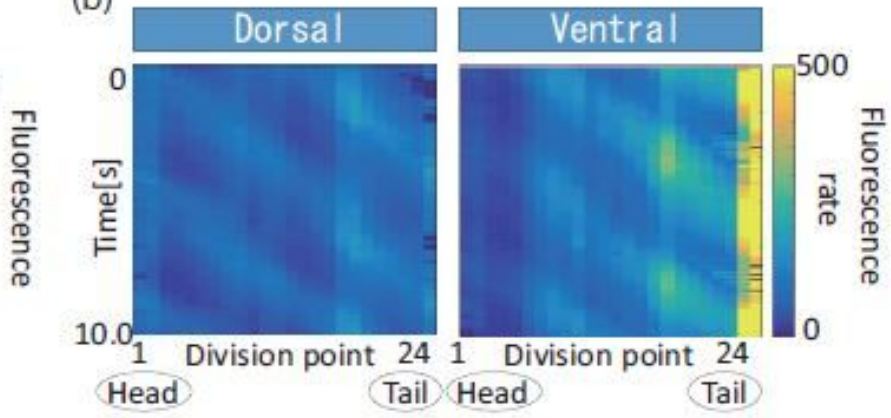

(d)

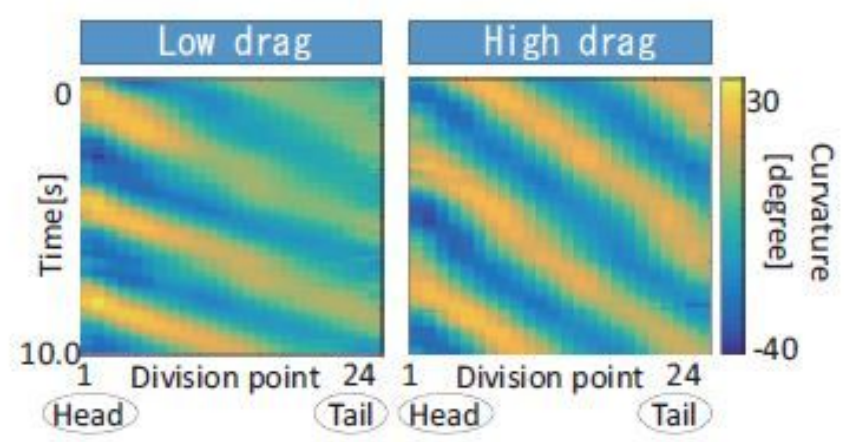

\section{Figure 4}

Measured fluorescence rates and curvature. (a) and (b) show examples of the fluorescence rate measured from an individual worm moving in low- and high-drag environments, respectively. The $\mathrm{x}$-axis denotes the division points, $y$-axis denotes time, and colour represents the fluorescence rate. (c) shows the fluorescence rate average of all individuals in the lowand high-drag environments at each division point. The $x$-axis denotes the division points, and the $y$-axis is the average fluorescence rate. Blue and green lines indicate dorsal and ventral sides, respectively. (d) shows the curvature of individuals moving through the low- and high-drag environments. (e) shows the phase difference between the curvature and difference components of the fluorescence rate averaged over individuals and division points. 
(a)

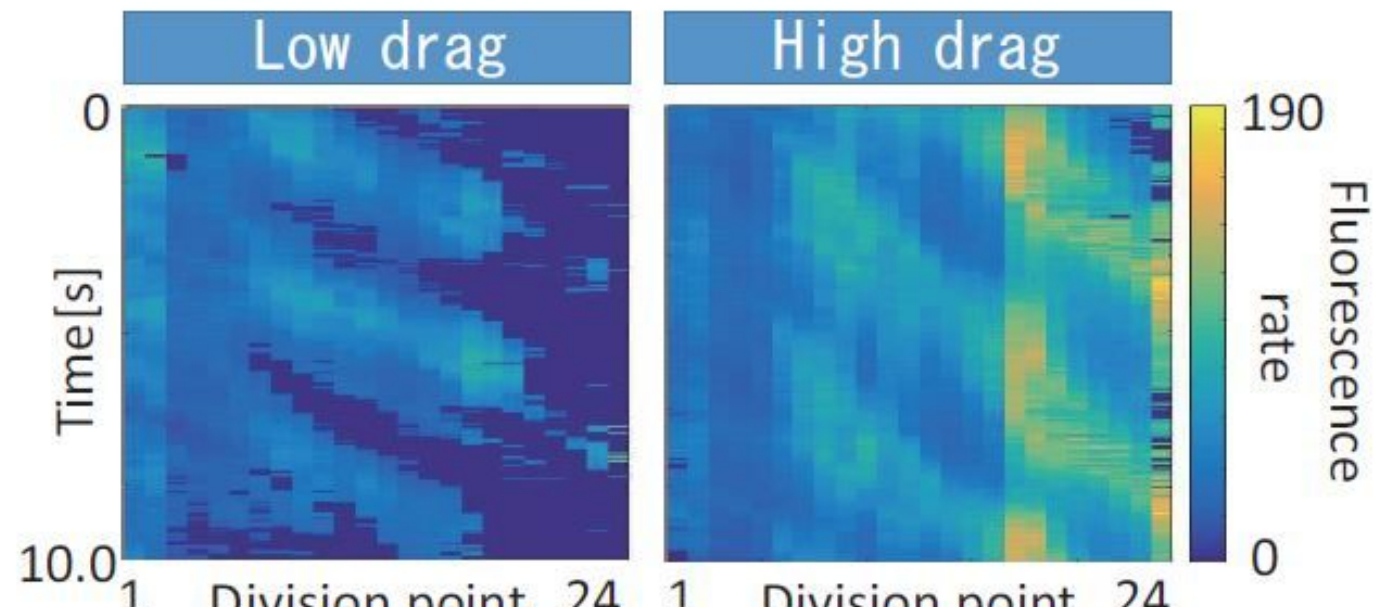

1 Division point $24 \quad 1$ Division point 24 Head Tail Head Tail

(b)

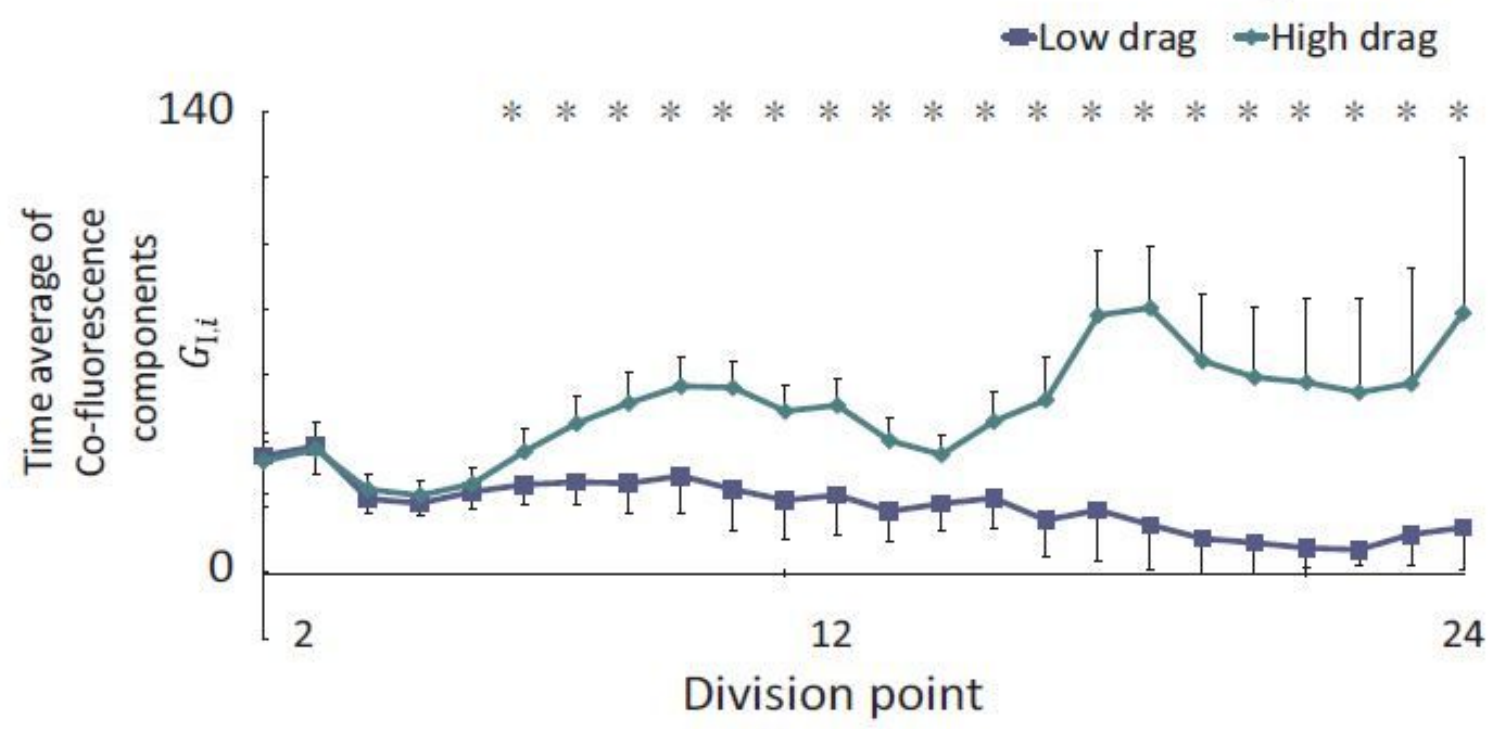

Figure 5

Co-fluorescence component. (a) shows an example of a co-fluorescent component. The x-axis denotes division points, $y$-axis denotes time, and colour represents the fluorescence rate. (b) shows the average of the co-fluorescent component over all individuals. The $x$-axis denotes the division points, and the $y$-axis represents the average co-fluorescence rate. 
(a)

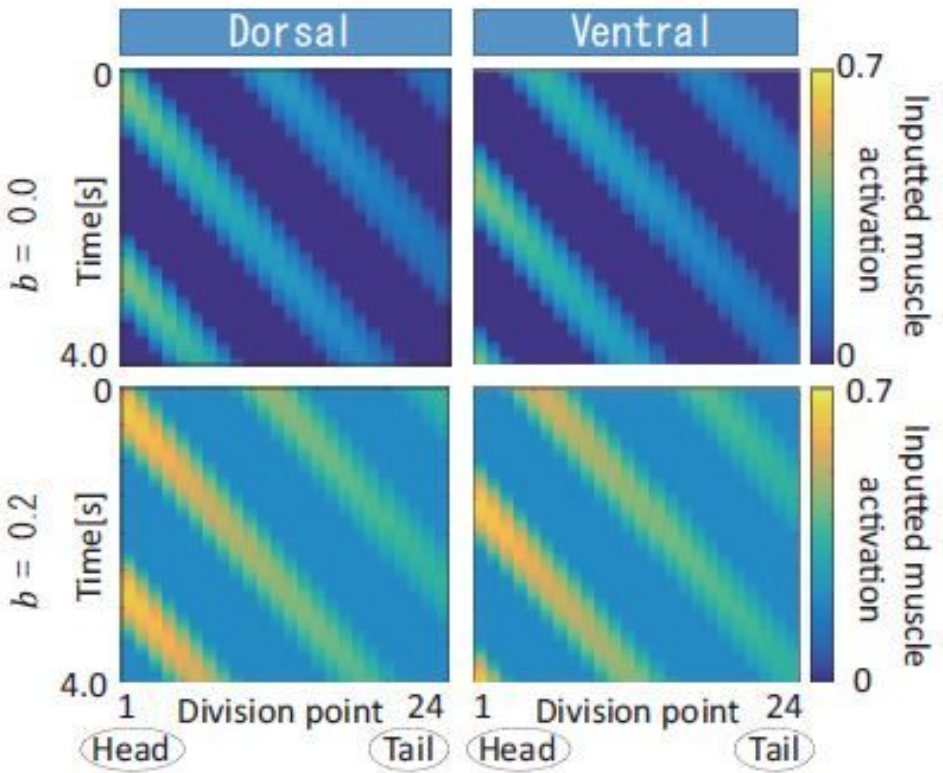

(c)

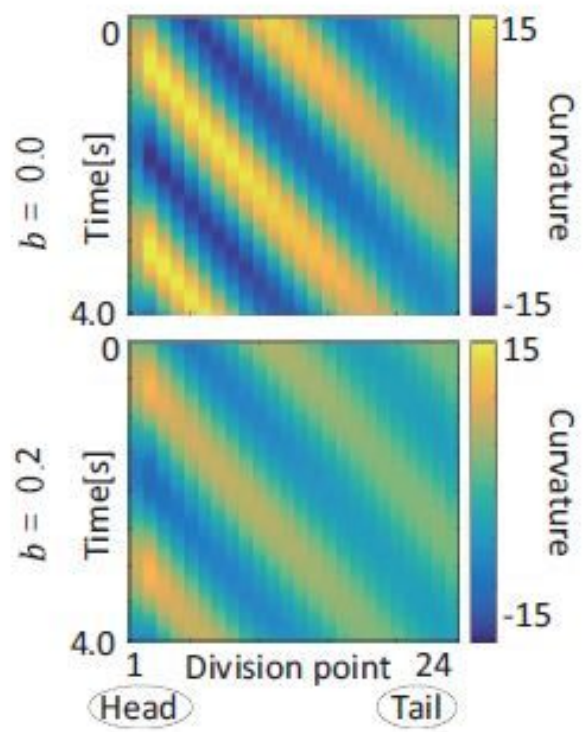

(b)

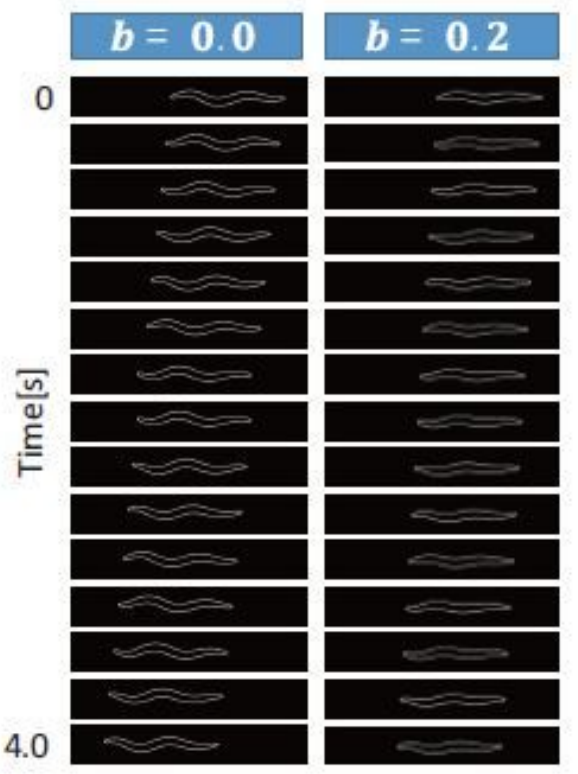

(d)

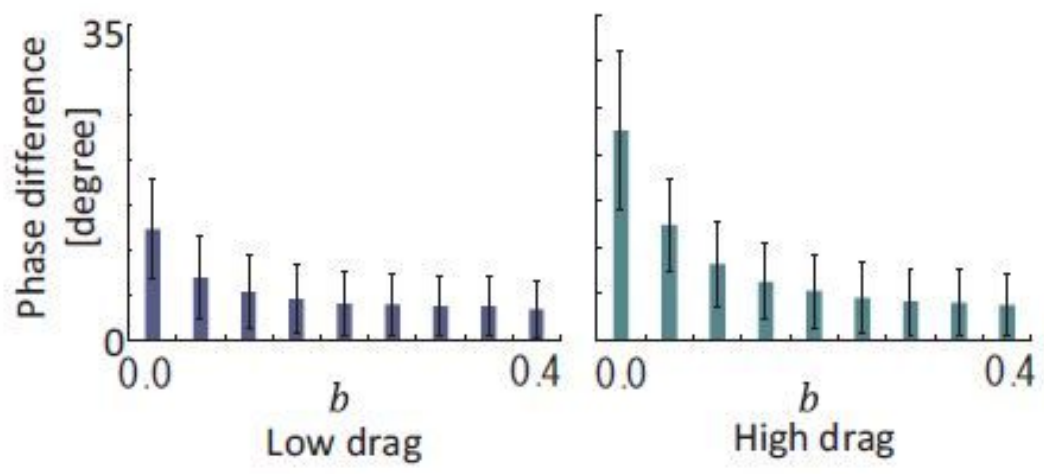

\section{Figure 6}

Simulation results of the body dynamics model. (a) shows the input of dorsal and ventral muscle activities with $b=0: 0$ and 0:2. The $x$-axis denotes the division points, $y$-axis represents time, and colour represents the inputted muscle activation. (b) shows the image obtained by sampling the generated motion at equal time intervals with $b=0: 0$ and $0: 2$. (c) shows the curvature $a t b=0: 0$ and $b=0: 2$. The $x$ axis denotes the division points, y-axis indicates time, and colour represents the curvature. (d) shows the individual average of the phase difference between the input muscle activity and curvature when $b=$ $0: 0 . .0: 4$. The $x$-axis denotes the co-contraction value, and the $y$-axis denotes the phase difference. 